POS PROCEEDINGS

\title{
Gamma Factory for CERN initiative - progress report
}

\section{Y. Dutheil*}

CERN, Geneva, Switzerland

E-mail: ydutheilecern.ch
A. Abramov ${ }^{1}$, S.E. Alden ${ }^{1}$, R. Alemany Fernández ${ }^{2}$, P.S. Antsiferov ${ }^{3}$, A. Apyan ${ }^{4}$, H. Bartosik ${ }^{2}$, E.G. Bessonov ${ }^{5}$, N. Biancacci ${ }^{2}$, J. Bieroń ${ }^{6}$, A. Bogacz ${ }^{7}$, A. Bosco ${ }^{1}$, R. Bruce ${ }^{2}$, D. Budker ${ }^{8,9}$, K. Cassou ${ }^{10}$, F. Castelli ${ }^{11}$, I. Chaikovska ${ }^{10}$, C. Curatolo ${ }^{12}$, P. Czodrowski ${ }^{2}$, A. Derevianko ${ }^{13}$, K. Dupraz $^{10}$, Y. Dutheil ${ }^{2}$, K. Dzierżęga $^{6}$, V. Fedosseev ${ }^{2}$, N. Fuster Martinez ${ }^{2}$, S.M. Gibson ${ }^{1}$, B. Goddard ${ }^{2}$, A. Gorzawski ${ }^{14,2}$, S. Hirlander ${ }^{2}$, J.M. Jowett ${ }^{2}$, R. Kersevan ${ }^{2}$, M. Kowalska², M.W. Krasny ${ }^{15,2}$, F. Kroeger ${ }^{16}$, D. Kuchler ${ }^{2}$, M. Lamont ${ }^{2}$, T. Lefevre ${ }^{2}$, D. Manglunki ${ }^{2}$, B. Marsh ${ }^{2}$, A. Martens ${ }^{10}$, J. Molson ${ }^{2}$, D. Nutarelli ${ }^{10}$, L.J. Nevay ${ }^{1}$, A. Petrenko ${ }^{17,2}$, V. Petrillo ${ }^{11}$, W. Płaczek ${ }^{6}$, S. Redaelli ${ }^{2}$, Y. Peinaud ${ }^{10}$, S. Pustelny ${ }^{6}$, S. Rochester ${ }^{18}$, M. Sapinski ${ }^{19}$, M. Schaumann ${ }^{2}$, R. Scrivens ${ }^{2}$, L. Serafini ${ }^{11}$, V.P. Shevelko ${ }^{5}$, T. Stoehlker ${ }^{16}$, A. Surzhykov ${ }^{20}$, I. Tolstikhina ${ }^{5}$, F. Velotti ${ }^{2}$, G. Weber $^{16}$, Y.K. Wu ${ }^{21}$, C. Yin-Vallgren ${ }^{2}$, M. Zanetti ${ }^{22,12}$, F. Zimmermann ${ }^{2}$, M.S. Zolotorev ${ }^{23}$ F. Zomer ${ }^{10}$

${ }^{1}$ Royal Holloway University of London Egham, Surrey, TW20 OEX, United Kingdom

${ }^{2}$ CERN, Geneva, Switzerland

${ }^{3}$ Institute of Spectroscopy, Russian Academy of Sciences, Troitsk, Moscow Region, Russia

${ }^{4}$ A.I. Alikhanyan National Science Laboratory, Yerevan, Armenia

${ }^{5}$ P.N. Lebedev Physical Institute, Russian Academy of Sciences, Moscow, Russia

${ }^{6}$ Marian Smoluchowski Institute of Physics, Jagiellonian University, Kraków, Poland

${ }^{7}$ Center for Advanced Studies of Accelerators, Jefferson Lab, USA

${ }^{8}$ Helmholtz, Institute, Johannes Gutenberg University, Mainz, Germany

${ }^{9}$ Department of Physics, University of California, Berkeley, USA

${ }^{10}$ LAL, Univ. Paris-Sud, CNRS/IN2P3, Université Paris-Saclay, Orsay, France

${ }^{11}$ Department of Physics, INFN-Milan and University of Milan, Milan, Italy

12 INFN-Padua, Padua, Italy

${ }^{13}$ University of Nevada, Reno, Nevada 89557, USA

${ }^{14}$ University of Malta, Malta

${ }^{15}$ LPNHE, University Paris Sorbonne, CNRS-IN2P3, Paris, France

${ }^{16}$ HI Jena, IOQ FSU Jena and GSI Darmstadt, Germany

${ }^{17}$ Budker Institute of Nuclear Physics, Novosibirsk, Russia

${ }^{18}$ Rochester Scientific, LLC, El Cerrito, CA 94530, USA

${ }^{19}$ GSI, Helmholtzzentrum für Schwerionenforschung, 64291 Darmstadt, Germany

${ }^{20}$ Braunschweig University of Technology and Physikalisch-Technische Bundesanstalt, Germany

${ }^{21}$ FEL Laboratory, Duke University, Durham, USA

22 University of Padua, Padua, Italy

${ }^{23}$ Center for Beam Physics, LBNL, Berkeley, USA 
The Gamma Factory (GF) initiative proposes to use partially stripped ion (PSI) beams as drivers of a new type of high-intensity and high-energy $(0.1-400 \mathrm{MeV})$ photon source. As part of the ongoing Physics Beyond Collider studies, initial beam tests were carried out in 2017 and 2018 at the SPS and LHC with partially stripped xenon and lead beams. This contribution discusses the results of these tests and the preparations for the next GF R\&D step: the proof-of-principle experiment at the SPS to study interaction of PSI beams with the laser light.

European Physical Society Conference on High Energy Physics EPS-HEP2019 10-17 July, 2019

Ghent, Belgium

${ }^{*}$ Speaker. 


\section{Key principles}

\subsection{Relativistic Doppler shift}

The Gamma Factory (GF) initiative relies on highly relativistic partially stripped ion (PSI) beams. The collision of photons propagating in the opposite direction to the PSI is the key phenomenon exploited by the GF initiative to produce high-energy high-flux photon beams.

In the laboratory reference frame:
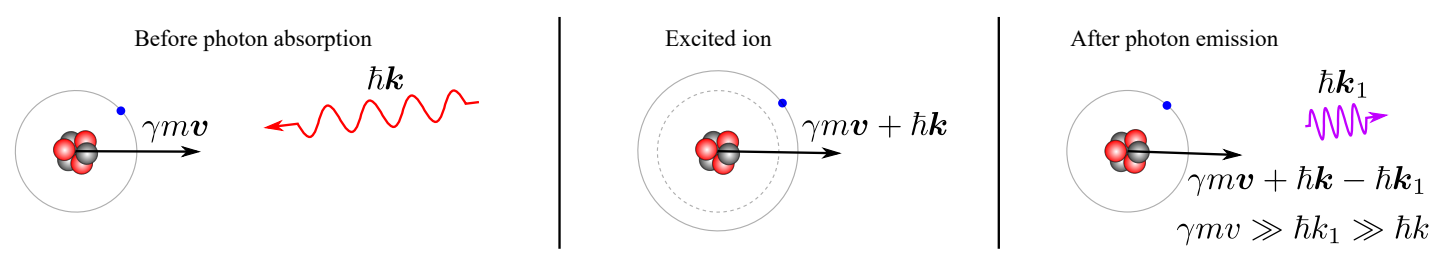

In the initial ion reference frame:
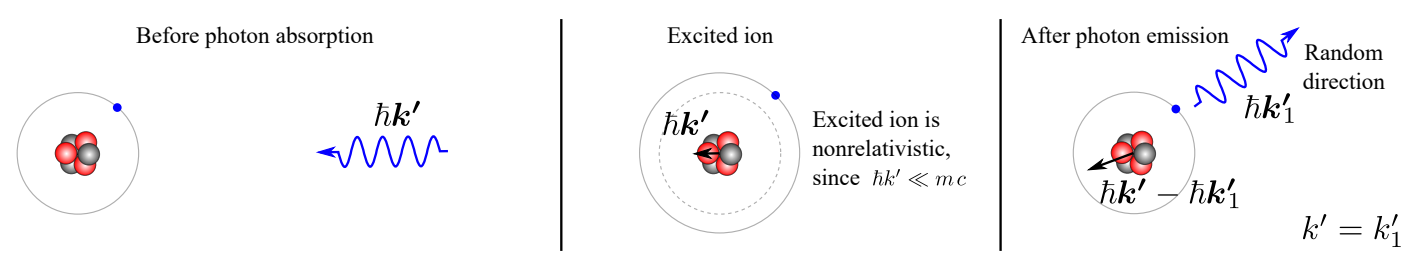

Figure 1: The process of photon scattering in the laboratory and rest reference frames.

Figure 1 shows the photon-ion collision process. Due to relativistic Doppler shift, the photon wavenumber in the ion reference frame $\left(k^{\prime}\right)$ is much higher than in the rest frame $(k)$ :

$$
k^{\prime}=(1+\beta \cos \theta) \gamma k \approx 2 \gamma k,
$$

with $\theta$ being the collision angle between the PSI and the photon directions in the laboratory reference frame, $\gamma$ - the Lorentz factor of the PSI and $\beta$ - the ratio of the PSI speed in the laboratory frame to the speed of light $c$. Equation 1.1 shows that the photon energy in the PSI frame is $2 \gamma$-times of that in the laboratory frame, in the approximation of a small collision angle $\theta$.

The collision of highly relativistic PSI with photons effectively acts as a photon-energy booster. The GF is already useful at this stage by allowing to excite deep electronic transitions.

\subsection{Resonant excitation}

Careful selection of collision angle, PSI and photon energies allows to target specific electronic transitions of the PSI. This resonant photon absorption by the PSI is likely, with typical cross sections of the order of gigabarns [1]. The process of direct scattering of photons on electrons is orders of magnitude less likely, with a typical cross section of the order of a barn.

The recoil of the PSI caused by the emission of the photon is small since $\hbar k^{\prime} \ll m c$, with $m$ the rest mass of the PSI (see Fig. 1). This effect still needs to be considered for the PSI beam dynamics but does not lead to an immediate loss of the ion. 


\subsection{Further energy boost for secondary photons}

Typically, a short-lived excited ion spontaneously emits a photon with the same energy as the absorbed one. However, in the laboratory reference frame the photon receives another Lorentz boost and the resulting photon wavenumber becomes:

$$
k_{1}=\left(1+\beta \cos \theta_{1}^{\prime}\right) \gamma k^{\prime} \approx 2 \gamma^{2}\left(1+\beta \cos \theta_{1}^{\prime}\right) k,
$$

with $\theta_{1}$ the angle of emission of the photon in the laboratory reference frame. For photons emitted with $\theta_{1}=0$ we find $k_{1} \approx 4 \gamma^{2} k$. The emitted photon energy depends on the emission angle but due to the Doppler shift the photons are preferentially emitted in a forward cone with an opening angle of $1 / \gamma$.

\subsection{Beam cooling}

The relaxation of the excited ion and subsequent photon emission changes the momentum of the PSI. The primary effect of such ultra-relativistic photon emission recoil is the reduction of the total ion momentum by a random value from zero to the maximum possible momentum of emitted gamma-photon (depending on the angle of photon emission). This introduces a friction force which reduces all components of ion momentum while the accelerating RF-cavities in a ring restore only the longitudinal component of the ion momentum. This leads to a gradual damping of transverse betatron oscillations or transverse cooling. Average energy extracted by the emission of photons grows with the ion energy as $\gamma^{2}$ which leads to a similar damping of longitudinal synchrotron oscillations since the average ion energy is maintained by the storage ring RF-cavities. This process of longitudinal cooling can be artificially enhanced by using a special spectral distribution of the laser pulse which would interact preferably with the ions above the central energy.

It is also possible to exploit a non-zero coupling between the position and energy of the PSI beam (dispersion) at the Interaction Point (IP) to achieve faster transverse cooling [6].

It is important to note that the emission of the photon $k_{1}^{\prime}$ is isotropic in the reference frame of the PSI in the cases considered here. The energy lost by the PSI is therefore random, which can also lead to heating of the longitudinal motion and the loss of the ion in some cases.

The method of laser cooling is well developed for low energy atomic beams, but it has never been applied to highly relativistic beams, such as those that can be accelerated in the SPS or the LHC.

\section{Physics opportunities}

The physics motivations of the GF initiative are numerous and they all have in common opening new research domains by making use of CERN's existing accelerator infrastructure, namely the SPS and LHC synchrotrons.

\subsection{Partially stripped ion beams}

High-energy beams of highly charged heavy ions, such as hydrogen-like lead $\left({ }^{208} \mathrm{~Pb}^{81+}\right)$, are of high interest for the atomic, molecular and optical physics community but have remained so far inaccessible [8]. 
Isoscalar nuclei, such as calcium or oxygen, contain the same number of protons and neutrons and are, therefore, best suited for the LHC electroweak precision measurements [9, 12]. For these relatively light species the luminosity is limited by the beam density. Partially stripped $\mathrm{Ca}$ or $\mathrm{O}$ ion beams could be quickly cooled in the SPS using the technique discussed Section 1.4 and provide high-brightness isoscalar beams to the LHC to maximise the collision luminosity.

Other applications of those atomic beams include the use of the bonded electron(s) for electronproton collision in the LHC or for plasma wakefield acceleration [1].

\subsection{Gamma-ray beams}

The photon frequency boost of $4 \gamma^{2}$ discussed Secion 1.3 can produce photon beams of up to $400 \mathrm{MeV}$ using the LHC.

Low-intensity gamma-ray sources in the $\mathrm{MeV}$ range exist in several countries with fluxes up to around $10^{10}$ photons per second. Those rely on inverse Compton scattering, which has a small cross section, and are limited by the available laser intensities and electron-beam currents. The GF photon flux is limited only by the available RF cavity power which makes possible to achieve the total photon-beam power in the order of $100 \mathrm{~kW}$. Such a high-intensity photon source could bring profit for a wide range of research, such as searching for dark matter or resolving the structure of atomic nuclei.

Photon collisions with centre-of-mass (CM) energies of the order of $100 \mathrm{keV}$ can be achieved by scattering a gamma-ray beam with a laser beam. Using the two rings of the LHC to produce the gamma-ray beams is considered for photon collisions with CM energies of up to $800 \mathrm{MeV}$.

\subsection{Secondary beams}

Another use of the GF gamma-ray beam is its interaction with an external target to produce secondary beams. With photon energy of up to 400 , the secondary beams of electrons and positrons but also muons become possible.

With careful selection of the photon energy produced by the GF, high-flux secondary beams of neutrons can be produced by making use of the excitation of the giant dipole resonance (GDR) of heavy nuclei.

\section{Progress}

The CERN accelerator complex is already producing and accelerating ions to high energies, in particular lead, xenon and argon, with plans to use oxygen in the coming years.

\subsection{PSI production}

The high efficiency production of the PSI is critical to the GF initiative. Detailed simulations to optimise the production of ${ }^{208} \mathrm{~Pb}^{81+}$ and ${ }^{208} \mathrm{~Pb}^{80+}$ using a single foil have been carried out. Measured efficiencies show an excellent agreement with predictions. This gives us strong confidence in improving the PSI production efficiency using dedicated strippers for each ion species in the future. 


\subsection{SPS and LHC tests}

Lifetime of PSI beams is limited by stripping through interaction with the rest gas. Measurement of those lifetimes is critical to improve the models and predict achievable GF figures. Since 2017 the production and storage of partially stripped ions have been experimentally studied at the SPS and LHC $[13,14,15]$.

Since then, the lifetimes of ${ }^{129} \mathrm{Xe}^{39+},{ }^{208} \mathrm{~Pb}^{81+}$ and ${ }^{208} \mathrm{~Pb}^{80+}$ beams have been measured in the SPS. In particular, the lifetime of the hydrogen-like lead-ion beam in the SPS was $600 \pm 30 \mathrm{~s}$. Better vacuum levels in the LHC allowed to reach for this beam the lifetime of $\sim 40 \mathrm{~h} \mathrm{[16].} \mathrm{Those}$ measurements allowed also to improve the numerical-simulation models and to make possible realistic predictions of the lifetimes for other PSI beams.

The efficiency of the LHC collimation system was also characterised with ${ }^{208} \mathrm{~Pb}^{81+}$ as it limits the maximum intensity safely achievable. However, the installation of new collimators in the dispersion suppression regions during the current shutdown is expected to mitigate this limit.

\section{Challenges}

The existing CERN accelerator complex has already demonstrated its capability to produce and accelerate the PSI beams. However, several aspects of the GF are being actively studied.

The source of photons to excite the PSI needs to provide high photon flux to maximise the fraction of the excited PSI and the repetition rate has to be of the order of $\mathrm{MHz}$ to interact with consecutive bunches of the PSI. Those two aspects are associated with high-power systems and can be satisfied by using a Fabry-Pérot (FP) resonant cavity. Laser systems using the FP cavity have already been developed and installed at the DESY and KEK electron beam storage rings by members of the GF collaboration [17, 18].

The modelling of interaction between the laser pulse and the PSI bunch poses new and unique challenges. Several modelling codes are being developed in parallel by different teams and using different numerical approaches to ensure the accuracy of predictions.

The dynamics of the PSI beam presents several new challenges. In the SPS, where the lifetime is limited by the residual gas in the ring with relatively poor vacuum, strong decay of the stored intensity needs to be accounted for.

In the LHC and in the scope of a continuous run of the GF, where the beam-cooling effects can be strong, it is critical to understand the dynamics of low emittance PSI beam that can become unstable due to collective effects. In such a case long-term equilibrium could be reached through the use of both cooling and heating.

\section{SPS Proof of Principle}

To demonstrate the feasibility of the most important aspects of the GF scheme a Proof of Principle (PoP) experiment is being proposed in the SPS [7]. The key objectives of this experiment are:

- Demonstrate integration and operation of a laser and a FP-cavity system in a hadron storage ring.

- Benchmark simulations of atomic excitation rates. 
- Develop a collision scheme and implement the required operational tools that demonstrate agreement of spatial and temporal properties of the ion and laser bunches; match laser spectrum to the atomic excitation width to demonstrate resonant excitation of an adequate fraction of the ion-bunch population; demonstrate reproducibility over many accelerator cycles.

- Demonstrate laser pulse and PSI bunch timing synchronisation.

- Measure and characterise the photon flux from the spontaneous emission and unfold the spectrum of emitted X-rays.

- Develop the PSI and photon-beam diagnostic methods.

- Demonstrate laser cooling of relativistic beams and investigate different approaches to cooling.

- Investigate the feasibility of relativistic atomic physics measurements.

\subsection{Experimental parameters}

Taking place in the SPS, the PoP uses the lithium-like lead ions ${ }^{208} \mathrm{~Pb}^{79+}$ at $\gamma \simeq 96$. The selected excited electronic transition is $2 s_{1 / 2} \rightarrow 2 p_{1 / 2}$ with a transition energy of approximately $231 \mathrm{eV}$. The proposed photon source consists of the FP cavity with photons of the energy at $1.2 \mathrm{eV}$ and with the round-trip frequency of $40 \mathrm{MHz}$ capable of colliding with the consecutive PSI bunches of the SPS. The cavity is intersecting the PSI beam axis at an angle of $2.6^{\circ}$ for the mirrors to remain outside the reserved SPS aperture. The lifetime of the excited $2 p_{1 / 2}$ state results in a decay length of the excited PSI in the laboratory reference frame of about $2 \mathrm{~m}$.

The main criteria to select a location on the SPS ring for the GF PoP experiment are:

- no major changes can be made to the SPS lattice (main magnets, key functional elements);

- the installed equipment must be compatible with the aperture required for the fixed-target and LHC beams;

- the radiation levels (prompt and residual) should be as low as possible around the laser electronics;

- a location with strong bumper magnets is preferred for steering the beam at high energy since the lattice correctors of the SPS are weak and we need a good control of the PSI beam to align it to the laser axis.

The location selected is situated immediately after the SPS long straight section 6, where the two missing dipoles of the dispersion-suppression region provide around $13 \mathrm{~m}$ of free space. Geographically, it is located on the main CERN site and close to the ATLAS experiment.

The main advantage of this location is an empty side tunnel (TI8) that was used for positron transfer to the LEP. It is envisioned to place the sensitive seed laser and the synchronisation equipment in this side tunnel to shield it from the high-radiation environment of the SPS.

\subsection{Observables}

It is crucial for the achievement of the PoP goals to list the observables and to model their expected behaviour. In particular, the accuracy, precision and dynamic range are considered [7].

To detect and measure the PSI excitation a scintillation screen is considered. The screen placed $7 \mathrm{~m}$ downstream of the IP absorbs the X-rays emitted by the excited ions. Simulated distributions of the produced X-ray photons were used to model the expected number of scintillation photons. In 
the nominal conditions a total of $3 \times 10^{13}$ photons $/ \mathrm{s}$ are produced in the visible range. Considering the usual geometry, the total flux on a camera would be around $2 \times 10^{11}$ photons/s, which is one order of magnitude above the sensitivity limit of the standard digital camera [20].

To measure and quantify the longitudinal cooling speed and strength we plan to use the existing SPS wall current monitor [21]. The transverse beam-cooling will be measured with the existing transverse-beam-size monitor based on beam gas ionisation [22].

\section{LHC scenarios}

The successful completion of the PoP objectives will grant a solid basis to the next generation of the GF experiment and give access to several of the physics opportunities discussed Section 2. In particular, several scenarios using the current LHC have been discussed within the GF collaboration.

The first scenario produces high-energy photons in the LHC by exciting the $1 s_{1 / 2} \rightarrow 2 p_{1 / 2}$ transition of the $\mathrm{Xe}^{53+}$ PSI. The estimated transition energy is estimated around $31 \mathrm{keV}$ [23] which gives photons of up to $185 \mathrm{MeV}$ in the laboratory reference frame. It requires laser photons at $5.2 \mathrm{eV}$ which could be produced with the same laser as the PoP experiment at its $5^{\text {th }}$ harmonic.

The second scenario maximises the photon flux. Using the $1 s_{3 / 2} \rightarrow 2 p_{3 / 2}$ transition with an energy of $3.9 \mathrm{keV}$, it produces photons at up to $26 \mathrm{MeV}$. The minimum laser photon energy is $0.58 \mathrm{eV}$ and could use the same laser as the PoP experiment, with a larger collision angle.

Many other scenarios are being discussed, both in the LHC and in SPS. In particular:

- production of photons in the LHC using ${ }^{208} \mathrm{~Pb}^{81+}$ to minimise the impact on the LHC physics programme;

- cooling of the calcium PSI beams in the SPS for high-luminosity collisions of these isoscalar nuclei in the LHC;

- direct collisions of ${ }^{208} \mathrm{~Pb}^{81+}$ with protons in the LHC to observe electron-proton and protonion collisions.

\section{Conclusions}

The GF initiative proposes an unconventional innovative use of the CERN accelerator complex to produce high-flux and high-energy photon beams. Opportunities in the HEP communities are numerous and extend to the broader physics community with the growth of the collaboration.

The last few years have seen passing several GF milestones. The acceleration and storage of the PSI beams was achieved in both the SPS and LHC. Software modelling tools are being developed to model both the PSI-photon interaction and the long-term behaviour of the PSI beams.

A PoP experiment in the SPS is being proposed for a possible start in 2023. The realisation of the PoP objectives would increase the momentum of the project and naturally pave the way towards the LHC scenario. 


\section{References}

[1] M. W. Krasny, “The Gamma Factory proposal for CERN,” arXiv:1511.07794 [hep-ex].

[2] E. G. Bessonov and K. J. Kim, "Radiative cooling of ion beams in storage rings by broadband lasers," Phys. Rev. Lett. 76 (1996) 431-434.

[3] M. Zolotorev and D. Budker, "Prospects in hydrogenic ions using high-energy accelerators," in Parity Violation in Atoms and Electron Scattering (B. Frois and M. A. Bouchiat, eds.), p. 364. World Scientific, 1999.

[4] U. Schramm et al., "Laser cooling of relativistic heavy ion beams," Conf. Proc. C0505161 (2005) 401.

[5] E. G. Bessonov and R. M. Feshchenko, "Stimulated Radiation Cooling," RuPAC Conf. Proc. (2008).

[6] I. Lauer et al., "Transverse Laser Cooling of a Fast Stored Ion Beam through Dispersive Coupling," Phys. Rev. Lett. 81 (1998) 2052-2055.

[7] M.W. Krasny et al., "Gamma Factory Proof-of-Principle Experiment,” CERN-SPSC-2019-031, CERN, Geneva, (2019).

[8] M. S. Safronova et al., "Search for New Physics with Atoms and Molecules," Rev. Mod. Phys. 90 (2018), no. 2 025008, 1710.01833.

[9] M. W. Krasny, S. Jadach, and W. Placzek, "The Femto-experiment for the LHC: The W-boson beams and their targets,” Eur. Phys. J. C44 (2005) 333-350, hep-ph/0503215.

[10] M. W. Krasny, F. Fayette, W. Placzek, and A. Siodmok, "Z-boson as "the standard candle" for high precision W-boson physics at LHC,” Eur. Phys. J. C51 (2007) 607-617, hep-ph/0702251.

[11] F. Fayette, M. W. Krasny, W. Placzek, and A. Siodmok, "Measurement of $\mathrm{M}_{W^{+}}-\mathrm{M}_{W^{-}}$at LHC," Eur. Phys. J. C63 (2009) 33-56, 0812.2571.

[12] M. W. Krasny, F. Dydak, F. Fayette, W. Placzek, and A. Siodmok, " $\Delta M_{w} \leq 10 M e V / c^{2}$ at the LHC: a forlorn hope?,” Eur. Phys. J. C69 (2010) 379-397, 1004.2597.

[13] S. Hirlaender et al., "Lifetime and Beam Losses Studies of Partially Strip Ions in the SPS $\left({ }^{129} \mathrm{Xe}^{39+}\right)$," in Proceedings, 9th International Particle Accelerator Conference (IPAC 2018): Vancouver, BC Canada, THPMF015, 2018.

[14] "Xenon beams light path to gamma factory," CERN Courier, November 2017, p. 7; cerncourier.com/xenon-beams-light-path-to-gamma-factory/.

[15] S. Charley, "LHC accelerates its first "atoms"," CERN News, 27 July, 2018, home. cern/news/news/accelerators/lhc-accelerates-its-first-atoms.

[16] M. Schaumann et al., "First Partially Stripped Ions in the LHC $\left({ }^{208} \mathrm{~Pb}^{81+}\right)$," in Proceedings, 10th International Particle Accelerator Conference (IPAC 2019): Melbourne, Australia, p. MOPRB055, 2019.

[17] S. Baudrand et al., "A High Precision Fabry-Perot Cavity Polarimeter at HERA,” JINST 5 (2010) P06005, 1005.2741.

[18] I. Chaikovska et al., "High flux circularly polarized gamma beam factory: coupling a Fabry-Perot optical cavity with an electron storage ring," Scientific Reports, 36569 EP, 2016.

[19] J. Bonis et al., "Non-planar four-mirror optical cavity for high intensity gamma ray flux production by pulsed laser beam Compton scattering off GeV-electrons," JINST 7 (2012) P01017, 1111.5833. 
[20] www.hamamatsu.com/eu/en/product/cameras/cmos-cameras/index.html

[21] T. Bohl and J. F. Malo, “The APWL Wideband Wall Current Monitor,” preprint CERN-BE-2009-006 CERN, Geneva (Feb, 2009).

[22] S. Levasseur et al., "Development of a rest gas ionisation profile monitor for the CERN proton synchrotron on a timepix3 pixel detector,' Journal of instrumentation 12 (2017), no. 02 C02050.

[23] W. R. Johnson and G. Soff, “The Lamb Shift in Hydrogen-like Atoms, $1 \leq Z \leq 110$ ” At. Data Nucl. Data Tables 33, 405âĂŞ446 (1985) 